1. Janzen, D. H. Coevolution of mutualism between ants and Acacia in Central America. Evolution 20, 249-275 (1966)

2. Hocking, B. Insect associations with the swollen-thorn acacias. Trans. R. Ent. Soc. Lond. 122, 211-255. 3. Knox, R. B., Marginson, R., Kenrick, J. \& Beattie, A. J. in Insects and the Plant Surface (eds Juniper, B. E. \& Southwood, T. R. E.) 295-307 (Arnold, London, 1986).

4. Del-Claro, K., Berto, V. \& Reu, W. Effect of herbivore deterrence by ants on the fruit set of an extrafloral nectary plant Qualea multiflora (Vochysiaceae). J. Trop. Ecol. 12, 887-792 (1996).

5. Niesenbaum, R. A. Linking herbivory and pollination: defoliation and selective fruit abortion in Lindera benzoin. Ecology 77, 2324-2331 (1996).

6. Koptur, S. in Insect-Plant Interactions (ed. Bernays, E.) 81-129 (CRC Press, Boca Raton, 1992)

7. Ross, J. H. An analysis of the African Acacia species: their distribution, possible origins and relationships. Bothalia 13, 389-413 (1981).

8. Young, T. P., Stubblefield, C. H. \& Isbell, L. A. Ants on swollen thorn Acacias: species coexistence in a simple system. Oecologia 109, 98-107 (1997).

9. Stone, G. N., Willmer, P. G. \& Nee, S. Daily partitioning of pollinators in an African Acacia community. Proc. R. Soc. Lond. B 263, 1389-1393 (1996).

10. Tybirk, K. Pollination, breeding system and seed abortion in some African acacias. Bot. J. Linn. Soc 112, 107-137 (1993)

11. Feinsinger, P. \& Swarm, L. A. How common are ant-repellent nectars? Biotropica 10, 238-139 (1978) 12. Prys-Jones, O. E. \& Willmer, P. G. The biology of alkaline nectar in the Purple Toothwort (Lathrea clandestina): ground level defence. Biol. J. Linn. Soc. 45, 373-388 (1992).

Acknowledgements. We thank D. Mafunde at Mkomazi Research Station for expert field assistance; T. Morgan and N. McWilliam for invaluable logistic back-up; and M. Ritchie and J. Graves for statistical advice. We thank the Royal Geographical Society, the Darwin Initiative and the British Council for financial support, as part of the Mkomazi Ecological Research Programme, and the Department of Wildlife of the Tanzanian Government.

Correspondence should be addressed to P.G.W. (e-mail: pgw@st-andrews.ac.uk).

\section{Evolution of genetic redundancy}

\section{Martin A. Nowak*, Maarten C. Boerlijst*, Jonathan Cooke $\nmid$ \& John Maynard Smith}

* Department of Zoology, University of Oxford, South Parks Road, Oxford OX1 3PS, UK

$\dagger$ National Institute for Medical Research, The Ridgeway, London NW7 1AA, UK $\ddagger$ School of Biological Sciences, University of Sussex, Brighton BN1 9QG, UK

Genetic redundancy means that two or more genes are performing the same function and that inactivation of one of these genes has little or no effect on the biological phenotype. Redundancy seems to be widespread in genomes of higher organisms ${ }^{1-9}$. Examples of apparently redundant genes come from numerous studies of developmental biology ${ }^{10-15}$, immunology $y^{16,17}$, neurobiology $y^{18,19}$ and the cell cycle $^{20,21}$. Yet there is a problem: genes encoding functional proteins must be under selection pressure. If a gene was truly redundant then it would not be protected against the accumulation of deleterious mutations. A widespread view is therefore that such redundancy cannot be evolutionarily stable. Here we develop a simple genetic model to analyse selection pressures acting on redundant genes. We present four cases that can explain why genetic redundancy is common. In three cases, redundancy is even evolutionarily stable. Our theory provides a framework for exploring the evolution of genetic organization.

There are an increasing number of observations demonstrating that experimental inactivation of certain genes has no apparent effect on the phenotype or fitness of an animal. In specific cases, it seems that the natural function of a gene can be taken over by another gene. Such a redundant genetic organization is sensible from an engineer's point of view: important functions require backup devices that can take over in case of failure. But can natural selection favour the emergence and stability of redundant genes?

Consider a population of animals in which some essential function can be performed by genes at either of two loci, $A$ and $B$. (We use the word 'function' to refer to an effect of a gene during development; thus two genes coding for different proteins can have the same function.) Non-functional alleles, $a$ and $b$, arise by mutation at rates $u_{a}$ and $u_{b}$ per generation; reverse mutations are ignored. For simplicity, we consider a haploid population, but the

\section{a}

Model 1

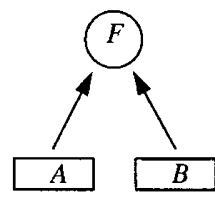

\begin{tabular}{|ll|}
\hline Gene & $F$ \\
$A$ & 1 \\
$a$ & 0 \\
$B$ & 1 \\
$b$ & 0 \\
\hline
\end{tabular}

\begin{tabular}{|ll|}
\hline Genotype & Fitness \\
$A B$ & 1 \\
$A b$ & 1 \\
$a B$ & 1 \\
$a b$ & 0 \\
\hline
\end{tabular}

b

Model 2

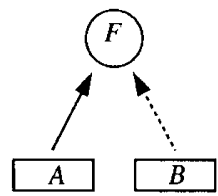

\begin{tabular}{|ll|}
\hline Gene & $F$ \\
$A$ & 1 \\
$a$ & 0 \\
$B$ & $h$ \\
$b$ & 0 \\
\hline
\end{tabular}

\begin{tabular}{|ll|}
\hline Genotype & Fitness \\
$A B$ & 1 \\
$A b$ & 1 \\
$a B$ & $h$ \\
$a b$ & 0 \\
\hline
\end{tabular}

C

Model 3

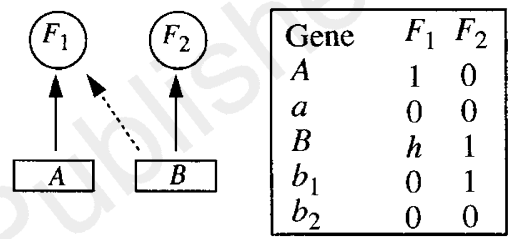

\begin{tabular}{|ll|}
\hline Genotype & Fitness \\
$A B$ & 1 \\
$A b_{1}$ & 1 \\
$a B$ & $h$ \\
other & 0 \\
\hline
\end{tabular}

Figure 1 Models of genetic redundancy. a, Model 1. If genes $A$ and $B$ perform function $F$ with equal efficacy, then redundancy does not persist. For unequal mutation rates, the gene with higher mutation rate will become extinct. b. Model 2. Redundancy can be evolutionarily stable if $B$ performs the function with lower efficacy, but also has a lower mutation rate. c, Model 3. Suppose $A$ and $B$ perform different functions, $F_{1}$ and $F_{2}$, but $B$ also performs function $F_{1}$, but with lower efficacy than gene $A$. The redundant organization for performing function $F_{1}$ is evolutionarily stable if the mutation rate that eliminates the cross-function in $B$ (but leaves its primary function unaffected) is lower than the mutation rate that inactivates $A$.

models can be extended to diploid populations and the conclusions remain essentially unchanged. There are four genotypes: $A B, A b, a B$ and $a b$. In each generation, random mating is followed by mutation and selection. Natural selection can maintain both genes if redundancy is only apparent, that is, if the $A B$ genotype is fitter than the other genotypes. Less obvious is the question of whether natural selection can maintain true redundancy in the sense that an individual with one of the two genes is as fit as an individual with both. Models 1-3 will address this question. Model 4 studies the consequence of developmental errors.

In model 1, we assume that both genes are equally effective, and that each can function perfectly on its own (Fig. 1a). The fitness of $A B, A b$ and $a B$ is one, while the fitness of $a b$ is zero. Let us first consider the case where the mutation rates in both genes are the same: $u_{a}=u_{b}=u$. The system admits a line of equilibria. All trajectories converge to this line. For small mutation rates, the maximum equilibrium frequency of $A B$ is approximately $1-2 \sqrt{ }(u / r)$, where $r$ is the recombination rate between the two loci. Thus a large proportion of individuals can carry functional alleles for both genes.

There is, however, an important caveat. We have assumed that the mutation rates $u_{a}$ and $u_{b}$ are equal. But any small deviation from $u_{a}=u_{b}$ destroys the equilibrium line. If $u_{a} \neq u_{b}$ then model 1 does not admit any interior equilibrium, and redundancy does not survive $^{22}$. A simple way of understanding this result is as follows. At equilibrium, the rate at which deleterious genes arise by mutation must equal the rate at which they are removed by selection. Because 
only $a b$ individuals are removed selectively, the rates of removal of the two genes are equal: therefore the rates at which they arise by mutation must be equal.

If $u_{a}>u_{b}$, then $A$ will become extinct while $B$ will be fixed. But if mutation rates are very similar, this may take a long time. In model 1 , A declines as $\exp \left[-\left(u_{a}-u_{b}\right) T\right]$, where $T$ is the number of generations ${ }^{22}$. (This represents an upper limit.) For a mutation rate of $10^{-6}$ per gene per generation, and a $10 \%$ difference between $u_{a}$ and $u_{b}$, the average lifetime of redundancy is about $10^{7}$ generations. Therefore, a certain amount of redundancy in our genomes could be the consequence of recent gene duplication events. We note, however, that redundancy cannot only be a consequence of gene duplication because very different genes can also show overlapping redundancy.

Several authors have studied stochastic versions of model 1 and computed the time it takes for random drift to eliminate one of the two genes even if mutation rates are exactly equal ${ }^{23-28}$.

In model 2, we assume that the genes $A$ and $B$ perform the same function, but with slightly different efficacies (Fig. 1b). Suppose $A$ performs the function with an efficacy of one, while $B$ does it with a reduced efficacy, $h$. If both genes are present, the function is performed with the higher of the two efficacies; this is essentially a definition of redundancy. Thus the fitness of genotypes $A B$ and $A b$ is one, while the fitness of genotype $a B$ is $h$. The fitness of $a b$ is zero. Unexpectedly, this can lead to a stable equilibrium with both genes $A$ and $B$ maintained in the population, provided that the mutation rate in $A$ is higher than in $\mathrm{B}$. Redundancy is maintained because gene $B$, with the lower efficacy, also has a lower mutation rate, and is maintained by selection in $a$ genotypes. In this case, $B$ is fully redundant in the sense that its inactivation has no effect on fitness, whereas deletion of $A$ causes a small reduction in fitness. A stable equilibrium is also possible if the fitness of $A b$ is higher than the fitness of $A B$, which in turn has a higher fitness than $a B$. In this case, redundancy is even maintained at a cost.

Model 3 relates pleiotropy to redundancy (Fig. 1c). Pleiotropy implies that genes perform more than one specific function (for example, by being expressed at more than one time and place in the developing organism ${ }^{29}$ ). The idea is that redundancy between two genes occurs only with respect to a given function, while the genes are maintained by selection because of another, independent func-

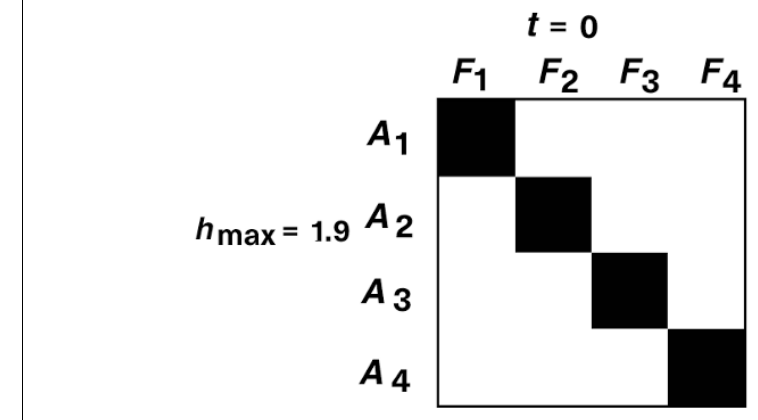

$t=100,000$

$t=500,000$
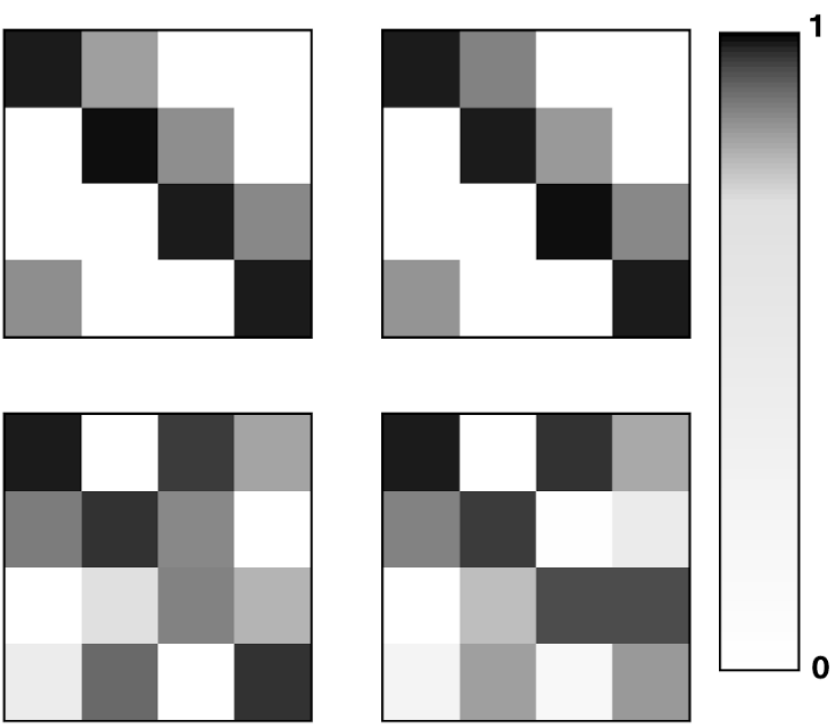

$h_{\max }=2.9$
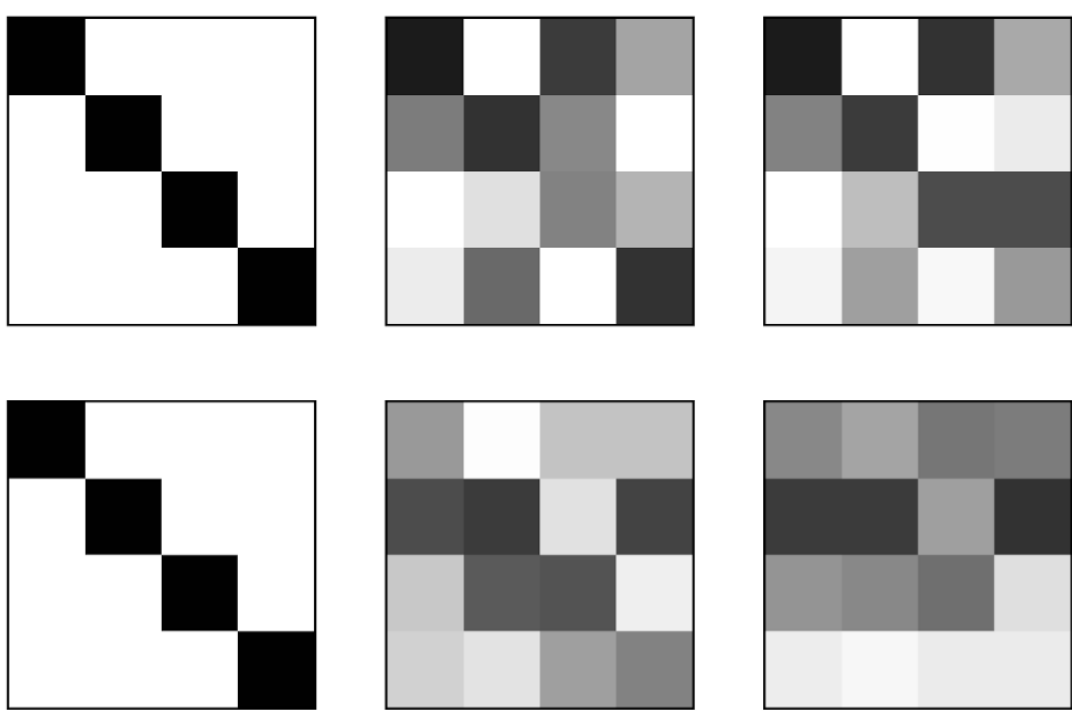

Figure 2 Complex redundancy-pleiotropy networks evolve if the mutation rate of complete inactivation of a gene is higher than the mutation rate of inactivating only one function of a gene while leaving other functions unaffected. Results from a stochastic computer simulation are shown. In each round, two individuals are chosen to reproduce, and their genes are recombined and mutated. Mutation can either inactivate a gene completely, inactivate one or more functions of a gene, or change the efficacies at which a gene performs a function. The fitness of the offspring is given by the product of the efficacies at which each function is performed, where each function is performed with the efficacy of the most efficient gene for this function. There are four loci, $A_{1}$ to $A_{4}$, and four functions, $F_{1}$ to $F_{4}$. Gene $i$ performs function $j$ with efficacy $h_{i j}$. Initially each gene performs one function with an efficacy of one. During the simulation, each gene can evolve to perform additional functions, but the sum over all efficacies is limited by $h_{\text {max }}$ for each gene: $\Sigma_{j} h_{i j}<h_{\max }$. For example, $h_{\max }=1.9$ means that a gene can perform one function with an efficacy of one and a second function with an efficacy of 0.9 . The figure shows the initial configuration, and the population averages of $h_{i j}$ after $t=100,000$ and $t=500,000$ generations for three different values of $h_{\text {max }}$. The population size is 20,000 . The mutation rate for complete inactivation is $u_{1}=0.001, u_{2}=0.0011, u_{3}=0.0012$ and $u_{4}=0.0013$ for loci $1-4$. A specific function is lost with mutation rate $u_{i} / 10$ and changed to random value between 0 and 1 (subject to $\Sigma_{j} h_{i j}<h_{\max }$ ) with mutation rate $u_{i} / 20$. 
tion. Redundancy arises as a consequence of 'functional overlap' between genes.

In the simplest case, there are two functions, $F_{1}$ and $F_{2}$, and two genes, $A$ and $B$. Suppose $A$ performs $F_{1}$ with an efficacy of one, while $B$ performs $F_{1}$ with a slightly reduced efficacy, $h$, and $F_{2}$ with an efficacy of one. Mutations in $A$ lead to the inactive variant $a$; the mutation rate is $u_{a}$. In the second locus, we consider two types of mutants: $b_{1}$ has lost the ability to perform $F_{1}$, but still performs $F_{2} ; b_{2}$ is completely inactive. The mutation rate from $B$ to $b_{1}$ is $u_{b 1}$.

The fitness of each variant is evaluated by assuming that each function is performed with the efficacy of the most efficient gene, and the overall fitness is the product of the efficacies at which the two functions are performed. Therefore genotypes $A B$ and $A b_{1}$ have a fitness of one, genotype $a B$ has fitness $h$, and all other genotypes have a fitness of zero.

Using the same framework as above, we find that a stable equilibrium with $A B$ is possible provided the mutation rate $u_{b 1}$ is smaller than $u_{a}$. Functional overlap (and therefore redundancy in performing function $F_{1}$ ) is stable if the mutation rate at which mutants are produced that have lost the functional overlap but still

a

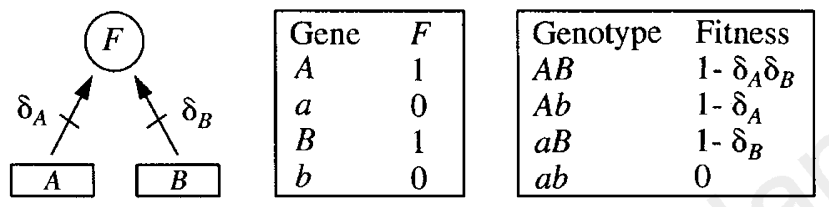

b

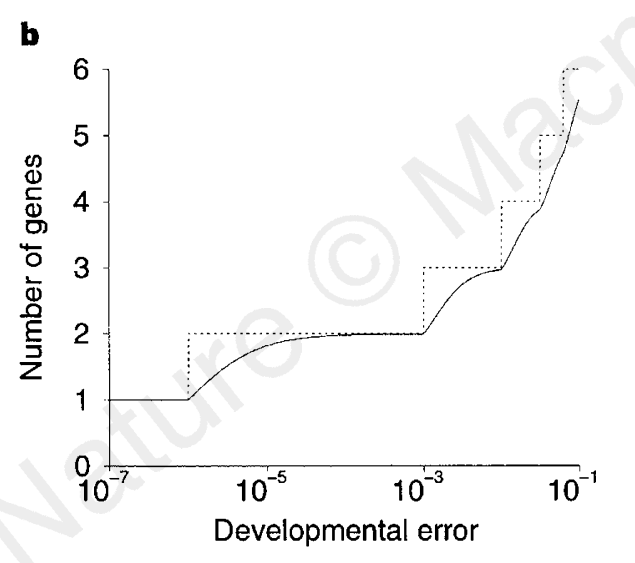

Figure 3 Developmental errors can provide selection pressure to maintain genetic redundancy. $\mathbf{a}$, Consider two genes $A$ and $B$ with mutation rates $u_{a}$ and $u_{b}$ and developmental error rates $\delta_{a}$ and $\delta_{b}$. Both genes are maintained in the population provided that $u_{a}<\delta_{b}$ and $u_{b}<\delta_{a}$. $\mathbf{b}$. The model can be extended to $n$ genes. Suppose all genes have mutation rate $u$ and developmental error rate $\delta$. The step function shows the maximum number of redundant genes that can coexist for a given development error rate $\delta$. The continuous function indicates the population average, $\Sigma_{i x_{i}}$, where $x_{i}$ is the frequency of genotypes with $i$ functional genes. The mutation rate is $u=10^{-6}$. maintain the original function is lower than the mutation rate of producing inactive mutants at the other locus $\left(u_{b 1}<u_{a}\right)$. This is plausible, as mutations that destroy all functions of a gene are likely to be more common than mutations destroying one function but leaving another unaffected. This is especially true if the two functions are very similar.

Models 2 and 3 show that true redundancy can be evolutionarily stable, but in both cases the relevant selection pressures are weak if the mutation rates are low. For weak selection pressures to counteract random drift the population size has to be large. In our case the population size has to exceed $1 / u$ for redundancy to be maintained.

Models 1-3 explore the maintenance of redundancy. We would also like to understand the origin of redundancy and extend the idea to a larger number of genes and functions. Figure 2 shows computer simulations based on a stochastic version of our model. The starting configuration is neither redundant nor pleiotropic: each function is performed by one gene, and each gene performs one function. During the simulation, mutations that lead to functional overlap arise spontaneously and can be favoured by selection. Genes evolve to perform additional functions. Redundancy is selected and can be fixed in the population if the mutation rate for complete inactivation of a gene is higher than the mutation rate for inactivating only a specific function of a gene. Final configurations often consist of complex redundancy-pleiotropy networks in which each function is performed by several genes and each gene performs several functions.

Finally, model 4 considers 'developmental errors' (Fig. 3). The transmission of information from the egg to the adult organism is subject to errors ${ }^{3}$. Let us therefore consider the possibility that a gene is intact in the germ line but fails to perform its function during development. We suggest that such developmental failure may arise either by somatic mutation, by errors in the origin and maintenance of cell differentiation (for example, in copying DNA methylation patterns), or through errors in cell-to-cell signalling ${ }^{30}$.

In terms of our model, we assume that two genes $A$ and $B$ perform the same function, but with probabilities $\delta_{a}$ and $\delta_{b}$ gene $A$ and $B$, respectively, fail to perform this function in the course of development. If both genes fail, the function is not performed and the animal does not survive.

In the absence of developmental error, the genotypes $A B, A b$ and $a B$ have a fitness of one, but taking into account developmental error, the average fitnesses of $A B, A b$ and $a B$ are respectively $1-\delta_{a} \delta_{b}$, $1-\delta_{a}$, and $1-\delta_{b}$. As before, the mutation rates to produce inactive genes $a$ and $b$ are $u_{a}$ and $u_{b}$. We find that the redundant genotype, $A B$, is stable if $u_{a}<\delta_{b}$ and $u_{b}<\delta_{a}$. Thus the mutation rate in each gene has to be smaller than the developmental error rate in the other gene. It is plausible that such developmental failures are more frequent than germline mutations: repeated rounds of cell replication provide increased probabilities for somatic mutation; errors can occur in the DNA methylation pattern and may result in incorrect cell differentiation; and interactions with other cells and response to signals can fail. Therefore we expect the error rate for normal expression of developmental genes, per individual ontogeny, to be higher than germline mutation rates. This is also supported by observations that routine examination of large numbers of embryos in each phase of development reveal spontaneous cases of obvious morphogenetic failure much in excess of germline mutation rates.

Model 4 suggests that redundancy should be more common in developmental genes that are expressed in specific spatio-temporal patterns in the body than in genes encoding for 'housekeeping' functions that are required in all cells (for example, essential metabolic enzymes). Somatic mutations or failures of gene expression that simply kill the cell in which they occur may have much less phenotypic effect than similar events that misguide subsequent developmental signals. Therefore the developmental error rate should be higher in genes that are not required in every cell. 
The model can be extended to more than two genes per function (Fig. 3b). An elegant result is obtained if we consider genes with similar mutation rate, $u$, and similar developmental error rate, $\delta$. The number of redundant genes that can be maintained per function is the largest integer less than $1+(\log u) /(\log \delta)$. For example, if the mutation rate is $u=10^{-6}$ and the developmental error rate is $\delta=10^{-3}$, selection can maintain up to three genes for a given function.

Model 1 shows that in situations where true redundancy is not evolutionarily stable, it may nevertheless take a long time until it is eliminated from the population, provided that mutation rates are small. Models 2 and 3 describe situations in which true redundancy can be maintained indefinitely. Model 3 can lead to complex redundancy networks in which each function is performed by several genes and each gene performs more than one function. Such networks are evolutionarily stable provided that random mutations are more likely to destroy all functions of a gene, rather than destroy just one function while leaving other functions unaffected. Model 4 introduces the concept of developmental errors, and shows that redundancy is evolutionarily stable provided that developmental error rates are larger than mutation rates in the germ line. According to model 4, redundancy should occur for those genes (or functions) that are under a high developmental error rate. The four models are not mutually exclusive; together they explain how mutation and selection can lead to redundant genetic organization.

\section{Methods}

Model 1. Consider a haploid population with genes at two loci, $A$ and $B$. Nonfunctional alleles, $a$ and $b$, arise at mutation rates $u_{a}$ and $u_{b}$. There are four genotypes, $A B, A b, a B$ and $a b$. The frequencies are $x_{1}, x_{2}, x_{3}$ and $x_{4}$, and the fitnesses are $f_{1}, f_{2}, f_{3}$ and $f_{4}$, respectively. In each generation there is mating (with recombination), followed by mutation and selection. Mating is described by the difference equations: $x_{1}^{\prime}=x_{1}+D, x_{2}^{\prime}=x_{2}-D, x_{3}^{\prime}=x_{3}-D$, and $x_{4}^{\prime}=x_{4}+D$. Here, $D=r\left(x_{2} x_{3}-x_{1} x_{4}\right)$, where $r$ is the recombination rate between the $A$ and $B$ loci, and $r$ is a number between 0 and 0.5 . Mutation is described by $x_{1}^{\prime}=x_{1}\left(1-u_{a}\right)\left(1-u_{b}\right), \quad x_{2}^{\prime}=x_{1}\left(1-u_{a}\right) u_{b}+x_{2}\left(1-u_{a}\right)$, $x_{3}^{\prime}=x_{1} u_{a}\left(1-u_{b}\right)+x_{3}\left(1-u_{b}\right)$, and $x_{4}^{\prime}=x_{1} u_{a} u_{b}+x_{2} u_{a}+x_{3} u_{b}+x_{4}$. Selection is described by $x_{i}^{\prime}=f_{i} x_{i} / f$, where $f=\Sigma_{i} x_{i} f_{i}$ denotes the average fitness of the population. Suppose both genes perform function $F$ with equal efficacy. We have $f_{1}=f_{2}=f_{3}=1$ and $f_{4}=0$. For exactly equal mutation rates, $u_{a}=u_{b}=u$, there is a line of equilibria given by $x_{1}=x_{2} x_{3} r(1-u) / u$. For unequal mutation rates, the gene with higher mutation rate will become extinct.

Model 2. This has the same framework as model 1 , but genes $A$ and $B$ perform function $F$ with different efficacies, $h_{a}$ and $h_{b}$. Let $h_{a}>h_{b}$. The genotype fitnesses are $f_{1}=f_{2}=h_{a}, f_{3}=h_{\mathrm{b}}$ and $f_{4}=0$. Redundancy can be evolutionarily stable if $B$ has a lower mutation rate than $A, u_{b}<u_{a}$. If $1-\left(h_{b} / h_{a}\right)>u_{a}>u_{b}\left[1+(1 / r)\left(h_{a}-h_{b}\right) / h_{b}\right]$ the equilibrium is $x_{1}^{*}=\left(1-x_{2}^{*}\right)$ $\times\left[h_{a}\left(1-u_{a}\right)-h_{b}\left(1-u_{b}\right)\right] /\left[\left(h_{a}-h_{b}\right)\left(1-u_{a}\right)\right], x_{2}^{*}=(1 / r)\left[u_{b} /\left(1-u_{b}\right)\right.$ $\times\left[h_{a}\left(1-u_{a}\right)-h_{b}\left(1-u_{b}\right)\right] /\left[h_{b}\left(u_{a}-u_{b}\right)\right], x_{3}^{*}=1-x_{1}^{*}-x_{2}^{*}$, and $x_{4}^{*}=0$. For low mutation rates, the equilibrium frequency of the redundant $A B$ genotype is approximately $x_{1}^{*} \approx 1-(1 / r)\left[u_{b} /\left(u_{a}-u_{b}\right)\right]\left(h_{a}-h_{b}\right) / h_{b}$. For example, if $h_{a}=1$, $h_{b}=0.99, u_{a}=1.1 \times 10^{-6}, u_{b}=10^{-6}$ and $r=0.5$, then the equilibrium frequency of $A B$ is about 0.8 .

This model can be expanded to $n$ genes with different mutation rates and different efficacies. The fitness of a particular genotype is given by the efficacy of the most efficient gene. If less efficient genes have lower mutation rates then stability of several redundant genes is possible. For a large number of genes, however, the conditions on efficacies and mutation rates become very restrictive.

Model 3. Consider two genes, $A$ and $B$, and two functions, $F_{1}$ and $F_{2}$. Gene $A$ performs function $F_{1}$ with efficacy $h_{a}$, and gene $B$ performs function $F_{1}$ with a lower efficacy $h_{b}$ and function $F_{2}$ with an efficacy of one. Mutations in $A$ lead to the inactive variant $a$; the mutation rate is $u_{a}$. Mutations in $B$ can either lead to variant $b_{1}$, which has lost the ability to perform function $F_{1}$ but still performs $F_{2}$, or to variant $b_{2}$, which is completely inactive; mutation rates are $u_{b 1}$ and $u_{b 2}$, respectively. Variant $b_{2}$ can also arise from $b_{1}$ at a mutation rate $u_{b 3}$. The redundant organization for performing function $F_{1}$, is evolutionarily stable if $u_{b 1}<u_{a}$. The analysis is similar to model 2 if $u_{b 2} \approx u_{b 3}$ : for low mutation rates, the equilibrium frequency of $A B$ is approximately $x_{1}^{*} \approx 1-(1 / r)\left[u_{b 1} /\left(u_{a}-u_{b 1}\right)\right]$ $\times\left(h_{a}-h_{b}\right) / h_{a}$. For the same numerical values as model 2 , and assuming that $u_{b 1}$ is 10 times smaller than $u_{a}$, we find that the equilibrium frequency of $A B$ is 0.998. Pleiotropy facilitates redundancy.

Model 4. Consider two genes $A$ and $B$ with mutation rates $u_{a}$ and $u_{b}$ and developmental error rates $\delta_{a}$ and $\delta_{b}$. Mutation and selection are described by the difference equations $x_{1}^{\prime}=\left(1-\delta_{a} \delta_{b}\right)\left(1-u_{a}\right)\left(1-u_{b}\right) x_{1} / f, x_{2}^{\prime}=\left(1-\delta_{a}\right)$ $\times\left(x_{1} u_{b}+x_{2}\right) / f, x_{3}^{\prime}=\left(1-\delta_{b}\right)\left(1-u_{b}\right)\left(x_{1} u_{a}+x_{3}\right) / f, x_{4}^{\prime}=0$, where $f$ is such that $x_{1}^{\prime}+x_{2}^{\prime}+x_{3}^{\prime}=1$. In contrast to models $1-3$, recombination is not essential here. The equilibrium frequency of $A B$ is $x_{1}=1 /\left\{1+\left[u_{a}\left(1-\delta_{b}\right)\right] /\right.$ $\left.\left[\delta_{b}\left(1-\delta_{a}\right)-u_{a}\left(1-\delta_{a} \delta_{b}\right)\right]+\left[u_{b}\left(1-\delta_{a}\right)\right] /\left[\delta_{a}\left(1-\delta_{b}\right)-u_{b}\left(1-\delta_{a} \delta_{b}\right)\right]\right\}$. For small values of $u$ and $\delta$, we obtain $x_{1} \approx 1 /\left\{1+\left[u_{a} /\left(\delta_{b}-u_{a}\right)\right]+\left[u_{b} /\left(\delta_{a}-u_{b}\right)\right]\right\}$. Thus necessary conditions for a large $x_{1}$ are $u_{a}<\delta_{b}$ and $u_{b}<\delta_{a}$.

The model can be extended to $n$ genes. Suppose all genes have mutation rate $u$ and developmental error rate $\delta$. Let $x_{i}$ denote genotypes with $i$ genes $(i=0, \ldots, n)$. The population dynamics are $x_{n-k}^{\prime}=\left(f_{n-k} / f\right) \sum_{i=0}^{k}\left(\begin{array}{c}n-i \\ k-i\end{array}\right)$ $\times u^{k-i} x_{n-i}$, where $f_{j}=\left(1-\delta^{j}\right)(1-u)^{j}$ and $f$ is such that all frequencies add to one. The equilibrium can be solved recursively. An equilibrium with the genotype containing all $n$ redundant genes is possible if $f_{n}>f_{n-1}$. This leads to $n<1+(\log u) /(\log \delta)$.

Diploid models. Our results for haploid models also apply to diploid models. In diploid models, we distinguish four gametes, $A B, A b, a B$ and $a b$, which form nine zygotes: $A B / A B, A B / A b, A b / A b, A B / a B, a B / a B, A B / a b, A b / a b, a B / a b$ and $a b /$ $a b$. For each generation we assume that mutation acts on gamete frequency, then zygotes are formed, selection acts on zygotes, and finally new gametes are formed, including the possibility of recombination. In agreement with haploid model 1, we find that the case where all zygotes have high fitness except $a b / a b$ which has low fitness, does not lead to stable redundancy. Cases similar to models 2 and 3 give stable redundancy. Diploid models with developmental errors also give stable redundancy.

There are some additional cases that can lead to redundancy in diploid models. One such case was discovered by Brookfield: it assumes that the double heterozygote, $A B / a b$, is as fit as the wild type, $A B / A B$, but $A b / a b, a B / a b$ and $a b /$ $a b$ have low fitness ${ }^{1}$. In addition, stable redundancy is also possible for partial dominance where all homozygotes have high fitness, the double heterozygote has a lower fitness, the single heterozygotes have still lower fitness, and $a b / a b$ has lowest fitness.

Classification of redundancy. It is helpful to distinguish three types of genetic redundancy. (1) True redundancy ${ }^{1}$ denotes the situation where an individual with a redundant genotype, $A B$, is not fitter than one in which one of the redundant genes has been knocked out, $A b$. In model 2, $B$ is truly redundant, but $A$ is not. In cases with pleiotropy, 'true redundancy' implies that the fully redundant genotype is not fitter than a genotype where the pleiotropic function of one gene has been eliminated. (2) 'Generic redundancy' is the case when an $A B$ individual is only occasionally fitter than an $A b$ individual. This can be the consequence of rare developmental errors. Another possibility is that $A B$ is only fitter than $A b$ in some environments. (3) 'Almost redundancy' means than the redundant genotype $A B$ is always slightly fitter than any genotype where one of the redundant genes has been knocked out. Of course, the fitness difference should be small if the situation is to be regarded as one of redundancy. Several such examples have been discussed previously ${ }^{5}$.

Received 14 January; accepted 25 April 1997.

1. Brookfield, J. F. Y. Genetic redundancy. Adv. Genet. 36, 137-155 (1997).

2. Brookfield, J. F. Y. Can genes be truly redundant? Curr. Biol. 2, 553-554 (1992),

3. Tautz, D. Redundancies, development and the flow of information. BioEssays 14, 263-266 (1992).

4. Goldstein, D. B. \& Holsinger, K. E. Maintenance of polygenic variation in spatially structured populations. Evolution 46, 412-429 (1992).

5. Thomas, J. H. Thinking about genetic redundancy. Trends Genet. 9, 395-399 (1993).

6. Dover, G. A. Evolution of genetic redundancy for advanced players. Curr. Opin. Genet. Dev. 3, $902-$ 910 (1993).

7. Pickett, F. B. \& Meeks-Wagner, D. R. Seeing double: appreciating genetic redundancy. Plant Cell 7, $1347-1356$ (1995)

8. Bird, A. P. Gene number, noise reduction and biological complexity. Trends Genet. 11, 94-100 (1995).

9. O'Brien, S. J. On estimating function gene number in eukaryotes. Nature New Biol. 242, 52-54 (1973).

10. Kastner, P. et al. Nonsteroid nuclear receptors: what are genetic studies telling us about their role in real life? Cell 83, 859-869 (1995).

11. Rudnicki, M. A. et al. Inactivation of MyoD in mice leads to up-regulation of the myogenic HLH gene Myf-5 and results in apparently normal muscle development. Cell 71, 383-390 (1992). 
12. Saga, Y et al. Mice develop normally without tenascin. Genes Dev. 6, 1821-1831 (1992).

13. Yang, Y. et al. Functional redundancy of the muscle-specific transcription factors Myf5 and myogenin Nature 379, 823-825 (1996)

14. Joyner, A. L. et al. Subtle cerebellar phenotype in mice homozygous for a targeted deletion of the En-2 homeobox. Science 251, 1239-1243 (1991).

15. Laney, J. D. \& Biggin, M. D. Redundant control of Ultrabithorax by zeste involves functional levels of zeste protein binding at the Ultrabithorax promoter. Development 122, 2303-2311 (1996)

16. Schorle, H. et al. Development and function of T cells in mice rendered interleukin-2 deficient by gene targeting. Nature 352, 621-624 (1991).

17. Taniguchi, T. Cytokine signaling through nonreceptor protein tyrosine kinases. Science 268, 251-255 (1995).

18. Steindler, D. A. et al. Tenascin Knockout Mice: Barrels, Boundary Molecules, and Glial Scars. J. Neurosci. 15, 1971-1983 (1995).

19. Pekny, M. et al. Mice lacking glial fibrallary acidic protein display astrocytes devoid of intermediate filaments but develop and reproduce normally. EMBO J. 14, 1590-1598 (1995).

20. Reed, S. I. G1-specific cyclins in search of an S-phase promoting factor. Trends Genet. 7, 95-99 (1991)

21. Roche, S. et al. Requirement for Src family protein tyrosine kinases in G-2 for fibroblast cell division. Science 269, 1567-1569 (1995).

22. Fisher, R. A. The sheltering of lethals. Am. Nat 69, 446-455 (1935).

23. Christiansen, F. B. \& Frydenberg, O. Selection-mutation balance for two nonallelic recessive producing an inferior double homozygote. Am. J. Hum. Genet. 29, 195-207 (1977).

24. Bailey, G. S. et al. Gene duplication in tetraploid fish: model for gene silencing at unlinked loci. Proc Natl Acad. Sci. USA 75, 5575-5579 (1978).

25. Allendorf, F. W. Protein polymorphism and the rate of loss of duplicate gene expression. Nature 272, $76-78(1978)$

26. Kimura, M. \& King, J. L. Fixation of a deleterious allele at one of two duplicate loci by mutation pressure and random drift. Proc. Natl Acad. Sci. USA 76, 2858-2861 (1979).

27. Li, W.-H. Rate of gene silencing at duplicate loci: a theoretical study and interpretation of data from tetraploid fish. Genetics 95, 237-258 (1980)

28. Ohta, T. Time for spreading of compensatory mutations under gene duplication. Genetics 123, 579584 (1989).

29. Li, X. \& Noll, M. Evolution of distinct developmental functions of three Drosophila genes by acquisition of different cis-regulatory regions. Nature 367, 83-87 (1994).

30. Krakauer, D. C. \& Pagel, M. Selection by somatic signals. Phil. Trans. R. Soc. Lond. B 351, 647-658 (1996).

Acknowledgements. We thank D. Krakauer and K. Sigmund for discussion. This work was supported by the Wellcome Trust (M.A.N.) and the European Community (M.C.B.)

Correspondence should be addressed to M.A.N. (e-mail: martin.nowak@zoo.ox.ac.uk).

\section{Distinct cortical areas associated with native and second languages}

Karl H. S. Kim ${ }^{\star}$, Norman R. Relkin $\dagger$, Kyoung-Min Lee ${ }^{\star} \&$ Joy Hirsch ${ }^{\star} \dagger$

Department of Neurology, ${ }^{*}$ Memorial Sloan-Kettering Cancer Center,

1275 York Avenue, New York, New York 10021, USA

$\dagger$ Department of Neurology and Neuroscience, Cornell University Medical College, 1300 York Avenue, New York, New York 10021, USA

The ability to acquire and use several languages selectively is a unique and essential human capacity. Here we investigate the fundamental question of how multiple languages are represented in a human brain. We applied functional magnetic resonance imaging (fMRI) to determine the spatial relationship between native and second languages in the human cortex, and show that within the frontal-lobe language-sensitive regions (Broca's area) ${ }^{1-3}$, second languages acquired in adulthood ('late' bilingual subjects) are spatially separated from native languages. However, when acquired during the early language acquisition stage of development ('early' bilingual subjects), native and second languages tend to be represented in common frontal cortical areas. In both late and early bilingual subjects, the temporal-lobe languagesensitive regions (Wernicke's area) ${ }^{1-3}$ also show effectively little or no separation of activity based on the age of language acquisition. This discovery of language-specific regions in Broca's area advances our understanding of the cortical representation that underlies multiple language functions.

Indirect evidence for topographic specialization within the language-dominant hemispheres of multilingual subjects has been provided by clinical reports of selective impairments in one or more of several languages as a result of surgery involving the left perisylvian area ${ }^{4}$. Multilingual patients with complex partial seizure disorders of temporal lobe origin have been reported to shift from a primary to a second language together with ictal progression ${ }^{5}$. Different languages have also been selectively disrupted in polyglots by electrical stimulation of discrete regions of the neocortex of the dominant hemisphere ${ }^{6,7}$. Changes in the topography of background electroencephalogram (EEG) coherence obtained during translation tasks also suggest spatial separation of cortical regions involved in multiple languages ${ }^{8}$. Although these reports are consistent with the existence of spatially separate representations for each language, such functions have not been localized.

Silent, internally expressive linguistic tasks were performed in two languages by subjects who either acquired conversational fluency in their second languages as young adults ('late' bilinguals) or who acquired two languages simultaneously early in their development ('early' bilinguals) (Table 1). As Broca's and Wernicke's areas are known to perform central roles in human language functions ${ }^{1-3,9-12}$, we have focused our observations on these cortical areas.

The main findings for a typical 'late' bilingual subject (subject (A)) are shown in Fig. 1. The anterior language area is highlighted by the green box and shown expanded in the inset. Red indicates significant activity during the native language task (English), whereas yellow indicates activity associated with the second language task (French). Two distinct but adjacent centres of activation (+) separated by $\sim 7.9 \mathrm{~mm}$ were evident within the inferior frontal gyrus, suggesting that two specific regions served each of the two languages. In the posterior language area of the same subject (Fig. 2 ), the same tasks yielded centroids of activity with a centre-tocentre spacing of $1.1 \mathrm{~mm}$, less than the width of a voxel, suggesting that similar or identical cortical regions served both languages in this posterior area.

For all six late bilingual subjects, distinct areas of activation were observed for the native and second languages in Broca's area (Table $2 \mathrm{a}$ and Fig. 3). The separation between centroids of activity ranged from $\sim 4.5 \mathrm{~mm}$ to $9.0 \mathrm{~mm}$ within one slice, and the number of voxels for each language was similar for each subject. On the other hand, activity in Wernicke's area (Table $2 \mathrm{~b}$ ) showed centre-to-centre distances between the centre-of-mass centroids ranging from 1.1 to $2.8 \mathrm{~mm}$. The mean centroid distance between the anterior

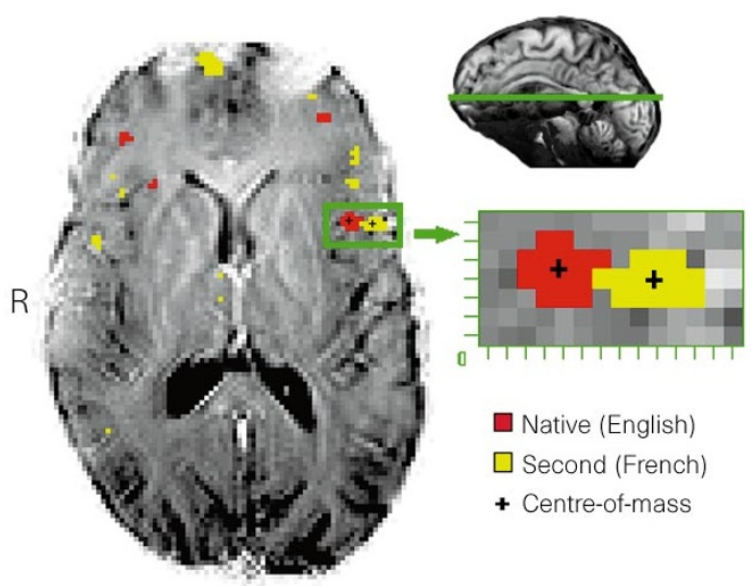

Figure 1 A representative axial slice from a 'late' bilingual subject $(A)$ shows all voxels that pass the multistage statistical criteria at $P \leq 0.0005$ as either red (native language) or yellow (second acquired language). An expanded view of the pattern of activity in the region of interest (inferior frontal gyrus, Brodmann's area 44 (refs 2, 3, 18), corresponding to Broca's area ${ }^{1-3}$ ) indicates separate centroids (+) of activity for the two languages. Centre-of-mass calculations indicate that the centroids are separated on this plane by $7.9 \mathrm{~mm}$. The green line on the upper right mid-sagittal view indicates the plane location. $R$ indicates the right side of the brain. 